\title{
Research on Photovoltaic Array's GMPPT Technology Based on Quantum-behaved Particle Swarm Optimization Algorithm
}

\author{
Lian Huang ${ }^{1, \text { a }}$, Pu Wang ${ }^{2}$, Suliang $\mathrm{Ma}^{1}$, Mingxuan $\mathrm{Chen}^{1}$, Jianwen $\mathrm{Wu}^{1}$, \\ ${ }^{1}$ School of Automation Science and Electrical Engineering, Beihang University, Beijing 100091 , \\ China; \\ ${ }^{2}$ Changping Power Supply Company, Beijing 102200, China; \\ aaoaottt@126.com
}

Keywords: photovoltaic array, partial shade, GMPPT, QPSO algorithm.

\begin{abstract}
On the problem of Global maximum power point tracking (GMPPT) caused by partially shaded in photovoltaic system, many scholars' search methods are always judged by their ability of static search, and dynamic performances when irradiance suddenly changes. But these methods neglect the dynamic performance when irradiance changes frequently. This article proceeded from the changes of maximum power point, which are according to the local approximate output characteristic in the process of illumination changes, analyzed the reason of the dead zone caused by the conventional optimization algorithms and combined optimization algorithm, and illustrated the necessity of the global optimization in the whole process. Then, according to the request of the whole and global optimization strategy, this article proposed a Quantum-behaved Particle Swarm Optimization (QPSO) to increase the diversity of the particles, the search velocity and convergence precision. Finally, the method was simulated by Maltab/SimScape and compared with the standard particle swarm optimization algorithm, showing the superiority of the algorithm in solving the problem of GMPPT caused by partially shaded.
\end{abstract}

\section{Introduction}

Distributed generation is one of the effective means which can make full use of clean and renewable energy to solve the problem of energy and the environment. Photovoltaic generation has played an important role in renewable energy in recent years for the sake of being safe and clean.

In order to increase the output power of PV array and improve the efficiency of photovoltaic power generation, global maximum power tracking technology has become the key point of the development of the distributed photovoltaic system under partial shadow condition.

This paper studies local shadow under the condition of output characteristics of photovoltaic power generation system evolution perspective. First, a mathematic model of the partial shadow under the condition of PV modules, as its multimodal optimization problems. The reason of failure on conventional optimization algorithms was analyzed. Then, on the basis of the optimization strategy and multimodal phenomenon, this paper introduce a behavior of quantum particle swarm optimization (QPSO) algorithm, in order to increase the particle diversity and improve the ability to jump out of local optimum, to speed up the search speed, effectively completed GMPPT function.

Finally, using the case analysis, and compared with the standard particle swarm algorithm, illustrate the behavior of quantum particle swarm optimization (PSO) algorithm is more suitable for solving local shelter under the condition of photovoltaic maximum power tracking problem, effectively improve the utilization efficiency of photovoltaic (PV) and power generation efficiency. 


\section{GMPPT Algorithm Implementation under Partially Shaded Condition}

\section{Failure Analysis of Conventional MPPT under Partially Shaded Condition}

In this paper, three kinds of illumination conditions of U-I curve and U-P curve is shown in Fig.1, for example, as shown in figure 1, shows that partial shadow GMPPT failure reason under the condition of the conventional method.

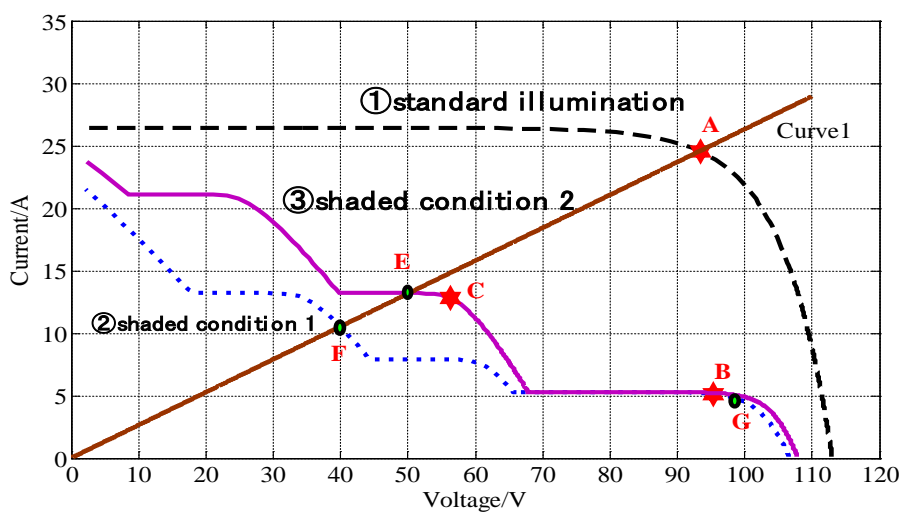

Fig.1 U-I curve of different illuminations

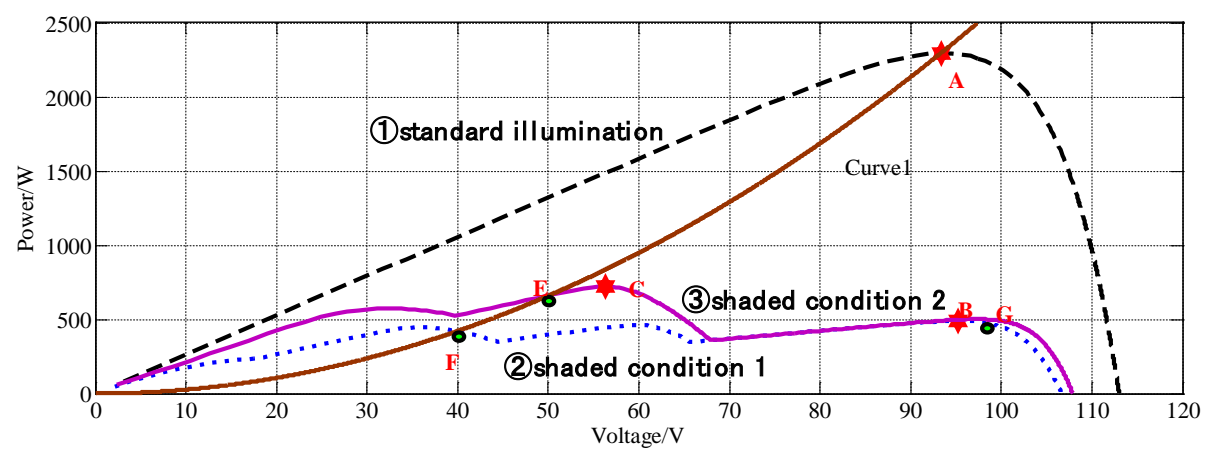

Fig.2 U-P curve of different illuminations

Curve 1 in the figure 1 shows the standard illumination conditions, the maximum power tracking point of the equivalent resistance load characteristic graph U-I and U-P. Point A is maximum power point of standard illumination condition. Point $\mathrm{B}$ is maximum power point under shaded condition 1 . Point $\mathrm{C}$ is maximum power point under shaded condition 2.

1) When illumination conditions changed from standard light conditions to shaded condition 2 , the corresponding power points from the point of $\mathrm{A}$ transformation to $\mathrm{E}$ at this point if the traditional optimization algorithms, such as observing disturbance, mountain climbing, etc., can find the optimal lighting condition of maximum power point C;

2) When illumination condition changed from standard conditions to cover A case, the corresponding power will change from $\mathrm{A}$ to $\mathrm{F}$, if at this time the traditional optimization algorithm, is difficult to trace to the maximum power point $\mathrm{B}$.

The classical optimization algorithm is adopted to solve the problem of local shadow GMPPT, namely multimodal problems. The traditional optimization algorithm is easy to failure, using the global search ability of the optimization algorithm, is the means to solve the problem.

\section{QPSO Algorithm in GMPPT}

According to conclusion discussed above, the shaded photovoltaic GMPPT problem of the traditional classical optimization algorithm couldn't satisfy the shielding requirements, it is necessary to establish a simple and easy method to realize the global search ability. GMPPT problem description such as formula (1).

$$
\left\{\begin{array}{l}
\max : P_{\text {out }}(D) \\
\text { s.t. } D \in[a, b]
\end{array}\right.
$$

As formula (1) shows, Pout (D) is output power of photovoltaic when D is the duty cycle of Boost converter, a is the lower limit for $\mathrm{D}$, $\mathrm{b}$ is expressed as the upper limit.QPSO algorithm 
implementation steps could be shown as follows:

Step1 Initialize parameters, and each particle in the particle swarm represents the current duty cycle Di (0), and record the optimal particle location Pi (0) = Di (0) and the global optimal duty cycle Gi (0).

Step2 According to the formula (2) calculating the average in the particle swarm optimal m(best) duty ratio

$$
\text { mbest }=\frac{1}{M} \sum_{i=1}^{M} P_{i}
$$

Step 3 According to the formula (3), update each particle represents the duty ratio Di (g)

$$
\begin{aligned}
D_{i}(g+1)= & \theta_{i} P_{i}(g)+\left(1-\theta_{i}\right) G(g) \\
& \pm \beta \mid \text { mbest }-D_{i}(g) \mid \ln \left(1 / u_{i}\right)
\end{aligned}
$$

Step4 Updated particle calculation fitness (photovoltaic cells output power), using the formula (3) update each particle own history compared optimal Pi (g) and the current global optimal duty cycle g (g).

$$
P_{i}(g)=\left\{\begin{array}{cc}
D_{i}(g) & f\left(D_{i}(g)\right)>f\left(P_{i}(g-1)\right) \\
P_{i}(g-1) & f\left(D_{i}(g)\right) \leq f\left(P_{i}(g-1)\right)
\end{array}\right.
$$

Step5 Analysis results whether meet the cut-off condition, if meet into Step6, otherwise make $g=$ $\mathrm{g}+1$ again to Step2 (this article sets the maximum number of iterations as the cut-off condition)

Step6 Output optimal duty ratio $\mathrm{G}$, terminate the optimization process.

\section{Simulation Verification}

\section{Simulation Conditions and Parameters}

To verify the algorithm discussed above, a simulation model is built under Matlab/Simulink. Simulation conditions and parameters are shown as Table 1.

Table 1 Three Scheme comparing

\begin{tabular}{cc}
\hline Parameters & Value \\
\hline Open-Circuit Voltage Uoc(V) & 37.67 \\
Short-Circuit Current Isc(A) & 8.81 \\
Maximum Power Pm(W) & 254.9 \\
Maximum Power Voltage Um(V) & 31.8 \\
Maximum Power Current Im(A) & 8.18 \\
\hline
\end{tabular}

\section{Results and Discussion}

Use quantum particle swarm optimization (QPSO) algorithm and standard particle swarm optimization (PSO) algorithm for the global power tracing experiment 20 times, respectively. The search results are shown in figure 3 and 4.
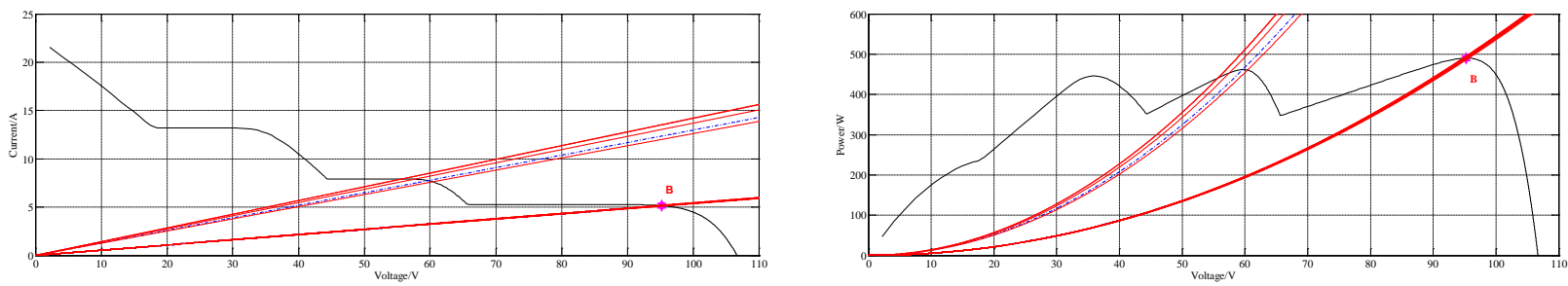

Fig.3 Algorithm results comparison between PSO and QPSO 


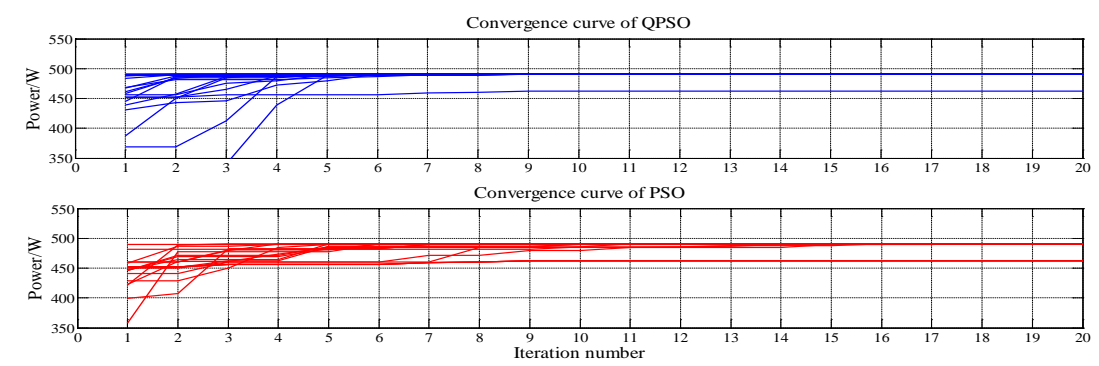

Fig.4 Global optimum curve comparison between PSO and QPSO

It can be seen from Figure3 and Figure 4 that the convergence rate of the quantum behaved particle swarm algorithm is much higher than that of the standard particle swarm optimization algorithm. According to the convergence curve of Figure4, we can see that the convergence rate of the quantum particle swarm algorithm based on the. So the superiority of quantum behaved particle swarm optimization is reflected in the rapidity and accuracy of the search.

\section{Summary}

This paper under the condition of partial shading, photovoltaic cells GMPPT problem research. First of all, through the analysis of partial shading of the impact of the current commonly used GMPPT algorithm analysis the failure reason, then constructed the quantum behaved particle swarm algorithm with strong global search ability, to achieve global maximum work rate tracking, conclusions are as follows:

1) The GMPPT problem is a typical multi peak optimization problem. The global search capability of the optimization algorithm, such as swarm intelligence optimization algorithm;

2) Introducing quantum behavior of improved particle swarm optimization algorithm, can well solve the multi peak problem and find excellent accuracy, rapidity and diversity are better than the standard particle swarm algorithm, applied to solve the problem of GMPPT under partial shading.

3) The quantum particle swarm (QPSO) algorithm proposed in this paper rely on only a few parameters and has fast global search capability which is suitable for GMPPT implementation.

\section{Acknowledgments}

This paper is Supported by the National Natural Science Foundation of China (51377007); Supported by the Specialized Research Fund for the Doctoral Program of Higher Education of China (20131102130006).

\section{References}

[1]. Koutroulis E, Blaabjerg F . A new technique for tracking the global maximum power point of PV arrays operating under partial-shading conditions[J] . IEEE Journal of Photovoltaics, 2012, 2(2): 184-190.

[2]. Paraskevadaki E V , Papathanassiou S A . Evaluation of MPP voltage and power of mc-Si PV modules in partial shading conditions[J] . IEEE Transactions on Energy Conversion, 2011, 26(3): 923-932.

[3]. Patel H, Agarwal V. Maximum power point tracking scheme for PV systems operating under partially shaded conditions[J]. IEEE Trans. on Industrial Electronics, 2008 , 55(4) : 1689-1698.

[4]. Walker G R , Sernia P C . Cascaded DC-DC converter connection of photovoltaic modules[J]. IEEE Trans. On Power Electronics, 2004, 19(4): 1130-1139.

[5]. Xiao W, Ozog N, Dunford W G. Topology study of photovoltaic interface for maximum power point tracking[J]. IEEE Trans. on Industrial Electronics, 2007, 54(3): 1696-1704. 